\title{
First-Principle Studies of Phonons III-N Compound Semiconductors in Wurtzite Structure
}

\author{
J. J. Zhang, G. J. Zhao, and X. X. Liang
}

\begin{abstract}
The phonon dispersion relations and density of states of the nitrides BN, AIN, GaN and InN in the wurtzite structure are investigated by using separately the local density approximation (LDA) and the generalized gradient approximation (GGA) within the framework of density functional theory. The results show that the highest optical phonon frequency at the Brillouin zone center decreases, but the phonon band gap increases, with increasing the cation/anion mass ratio. The frequencies calculated with GGA are lower than those with LDA. The GGA is more suitable for AIN and GaN, but the LDA for BN and InN.
\end{abstract}

Index Terms-Phonon dispersion, phonon density of states, III-N compound semiconductor, wurtzite structure.

\section{INTRODUCTION}

In recent years, the group III-nitride compound semiconductors have been attracting considerable attention, for their distinguished features. It is known that the nitrogen in III-N compounds forms rather short bonds with the trivalent metallic elements [1], and then the corresponding materials have a considerable hardness, high thermal conductivity and direct energy gap related to a substantial range from the visible to ultraviolet region of spectra. Consequently, nitride materials have shown a large impact on the optoelectronic devices in the deep ultraviolet [2] as well as on the electronic devices for high power, high-frequency operation. The nitride-based visible light emitting diodes are recently given more priority due to their application in solid state lightings [3], [4]. Current activities in optoelectronic devices have led to significant interest in studies of the structural and electronic properties in both wurtzite and zinc-blende phases of III-N semiconductors [5], [6]. The lattice dynamics of these materials have also been studied theoretically [7]-[11].

In present paper, the first-principle calculations for the lattice dynamics properties of $\mathrm{AlN}, \mathrm{GaN}, \mathrm{BN}$ and $\mathrm{InN}$ in wurtzite structure are performed with two different exchange- correlation functionals. The computed results by two different approaches are compared with each other as well as with the existing theories and experiments.

\section{THEORETICAL METHODS}

We have considered the III-N compound semiconductors in wurtzite structure and obtained the structural and

Manuscript received January 25, 2013; revised May 6, 2013

The authors are with the Department of Physics, Inner Mongolia University, Hohhot, China 010021 (e-mail: xxliang@imu.edu.cn). dynamical properties of the GaN, AlN, BN and $\mathrm{InN}$ by the first-principle calculation based on the density functional theory. The exchange-correlation potentials in the local density approximation (LDA) [12] and the generalized gradient approximation (GGA) [13] are separately used in the calculations. The optimum plane-wave energy cut-off has been tested carefully by keeping the total energy error less than 0.001 Hartree, and is determined as 45 Ryd for all these compounds. A $3 \times 3 \times 3$ Monkhorst-Pack mesh and ten special $k$ point have been used to perform the Brillouin zone (BZ) integration. The self-consistent convergence of the total energy is taken to be $10^{-6} \mathrm{eV} / \mathrm{atom}$, in order to obtain accurately the phonon dispersion curves. Moreover, a $4 \times 4 \times 4$ $q$ mesh is employed for the force constant calculation in the first BZ by interpolation. The whole calculation is carried out by using the Quantum ESPRESSO code [14], and the calculated results of the phonon frequencies and densities of states (DOS) are shown in Fig. 1 to Fig. 4.

\section{RESUlTS AND DISCUSSION}

To obtain the equilibrium lattice constant $a, c$ and the internal geometrical constant $u$, we fit the energy versus volume curves to the Murnaghan equation of state. Table I lists the calculated values of the structural parameters in this work. It is seen that our results are in good agreement with the previous theoretical and experimental values.

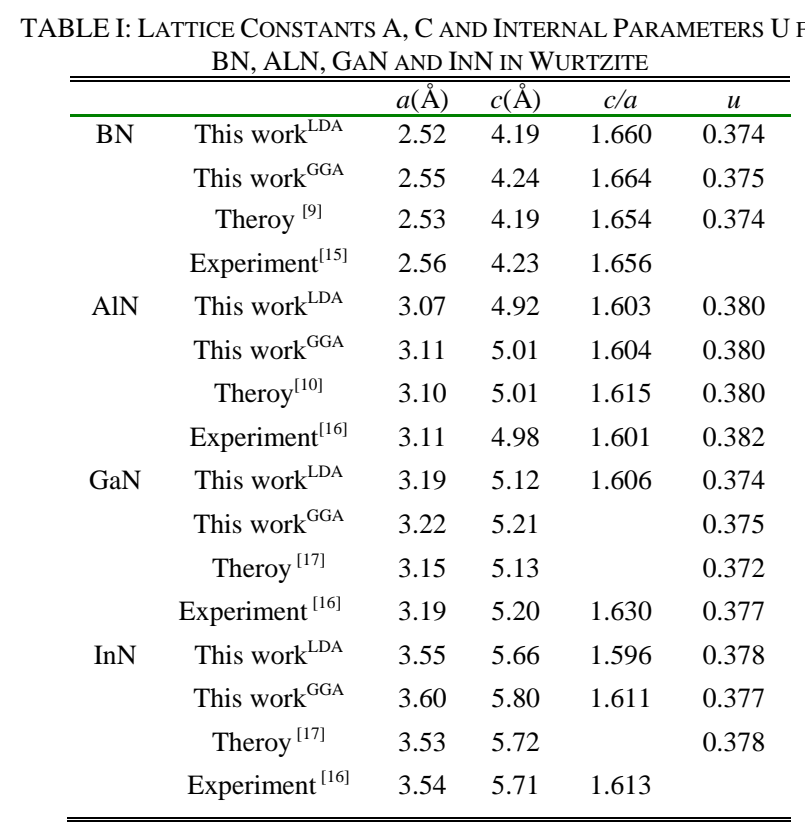

We have calculated the phonon dispersion curves and DOS of AlN, BN, GaN, InN of wurtzite structure by using 
the exchange-correlation potentials GGA and LDA, respectively, and the results are plotted in Figs. 1-4. Similar qualitatively dispersive curves and DOS of phonons for 4 different nitride semiconductors are obtained. It is expected that there are 12 branches of phonon frequency curves for every material, because a unit cell contains 4 atoms and then 12 degrees of freedom for wurtzite III-N compounds. The phonon modes degenerate at some high symmetry points of BZ. At $A$ point, only 4 phonon modes can be observed, but 8 modes called $2 A_{1}, 2 B_{1}, 2 E_{1}$ and $2 E_{2}$ respectively appear at the $\Gamma$ point. However, the quantitative characteristics of phonon frequency branches for the calculated materials are different from each other's upon the different cations.

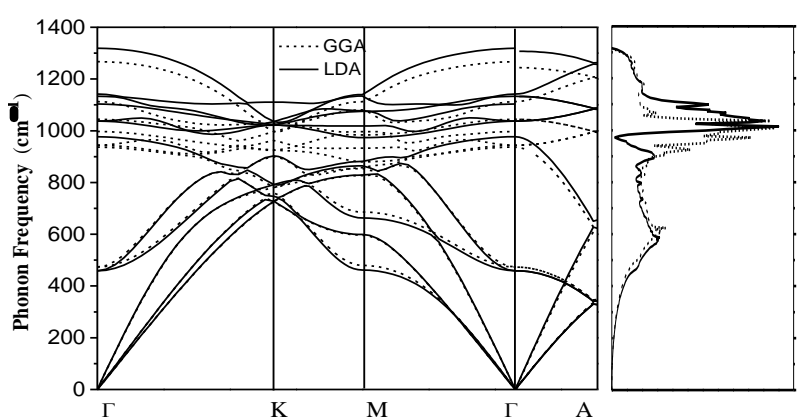

Fig. 1. Phonon dispersion curves (left) and DOS (right) of wurtzite BN.

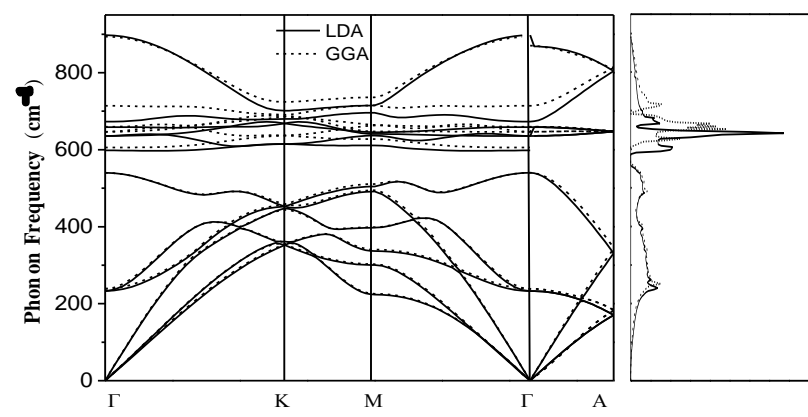

Fig. 2. Phonon dispersion curves (left) and DOS (right) of wurtzite AlN.

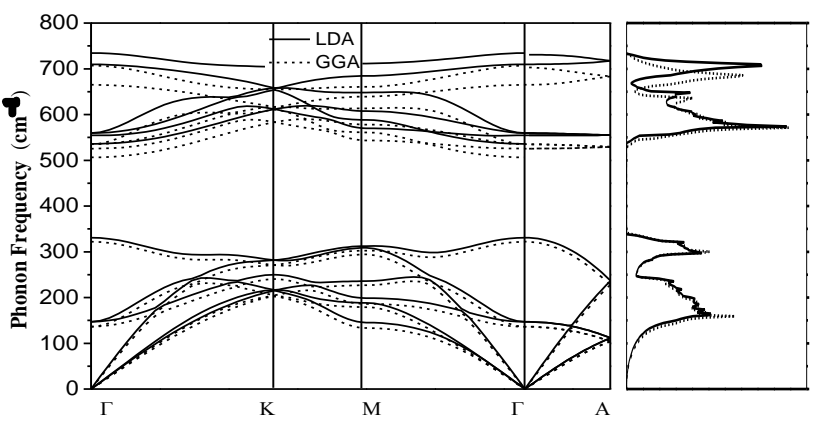

Fig. 3. Phonon dispersion curves (left) and DOS (right) of wurtzite GaN

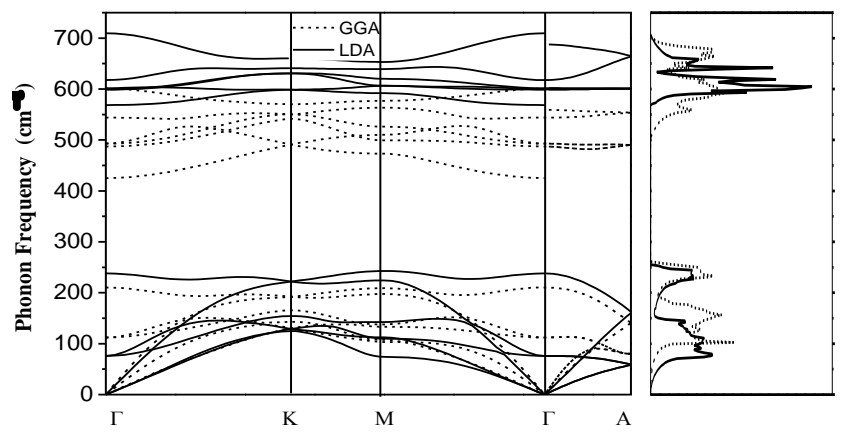

Fig. 4. Phonon dispersion curves (left) and DOS (right) of wurtzite InN.
For ease of understanding the mass influence to the frequency characteristic, we list the relative masses $m_{\mathrm{A}}$ of the cations, mass ratios $m_{\mathrm{A}} / m_{\mathrm{C}}$, and reduced masses $m_{\mathrm{A}} m_{\mathrm{C}} /$ $\left(m_{\mathrm{A}}+m_{\mathrm{C}}\right)$ in Table II. The anion mass $m_{\mathrm{C}}$ is same as 14.01 for the 4 calculated materials.

TABLE II: RELATIVE MASSES OF CATION AND ANION, MASS RATIOS AND REDUCED MASSES $\mathrm{M}_{\mathrm{A}} \mathrm{M}_{\mathrm{C}} /\left(\mathrm{M}_{\mathrm{A}}+\mathrm{M}_{\mathrm{C}}\right)$

\begin{tabular}{ccccc}
\hline \hline Material & BN & AlN & GaN & InN \\
\hline Cation mass $m_{\mathrm{A}}$ & 10.81 & 26.98 & 69.72 & 114.8 \\
$m_{\mathrm{A}} / m_{\mathrm{C}}$ & 0.77 & 1.93 & 4.98 & 8.19 \\
$m_{\mathrm{A}} m_{\mathrm{C}} /\left(m_{\mathrm{A}}+m_{\mathrm{C}}\right)$ & 6.10 & 9.24 & 11.67 & 12.49 \\
\hline \hline
\end{tabular}

It can be read from the figures that the characteristics of phonon frequencies are sensitive to the cation/anion mass ratios. Firstly, the highest frequency of optical phonons at the BZ center ( $\Gamma$ point) increases obviously with decreasing the cation mass and then the reduced mass in InN, GaN, AlN, BN order. Meanwhile, it is also seen in the DOS curves that there are the phonon band gaps between the acoustic and optical phonon branches in $\mathrm{InN}, \mathrm{GaN}$ and $\mathrm{AlN}$ because of the cation-anion mass mismatches, and the gap width increases with increasing the cation mass, consistent with the previous work [10]. However, the band gap can not be observed in BN whose cation-mass is closes to the anion-mass. Fig. 5 shows the widths of the phonon band gaps for the BN, AlN, GaN and InN. One can clearly see that the band gap expend non-linearly with enhancing cation/anion mass ratio. The ratio of the cation to anion mass in $\mathrm{BN}$ is slight lower than 1 , and then the gap can not be observed.

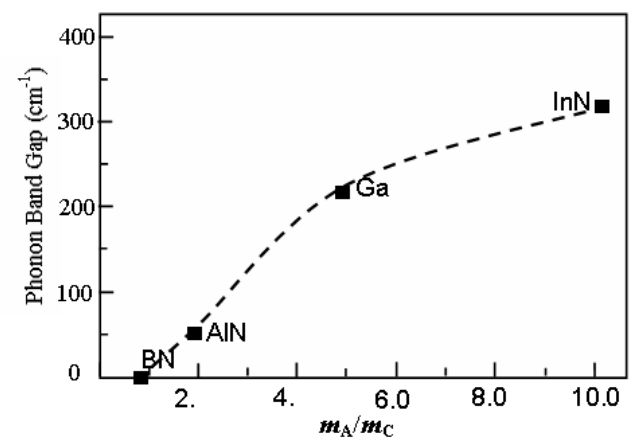

Fig. 5. Phonon ban gaps as functions of cation/anion mass ratio for BN, AlN, $\mathrm{GaN}$ and $\mathrm{InN}$.

Now we turn to analyze the scale of the LO-TO splitting in the phonon spectrums. The calculated relative splitting $\left(\omega_{\mathrm{LO}}-\omega_{\mathrm{TO}}\right) / \omega_{\mathrm{TO}}$ for $E_{1}\left(A_{1}\right)$ mode are $0.21(0.25)$ in $\mathrm{BN}, 0.37$ (0.46) in AlN, 0.33(0.36) in $\mathrm{GaN}$ and $0.26(0.29)$ in $\mathrm{InN}$, respectively. It may be seen that the greater the relative value of splitting, the more LO mode dispersion is evident. It is also seen that there exist a deviation of the frequency value around $\Gamma$ along the direction of $M-\Gamma$ and $A-\Gamma$ due to the anisotropy of the wurtzite structure. The ratio $\left[\omega_{\mathrm{TO}}\left(E_{1}\right)-\omega_{\mathrm{TO}}\left(A_{1}\right)\right] / \omega_{\mathrm{TO}}\left(E_{1}\right)$ is usually used to measure the anisotropy of materials, for which our calculated values are $0.05,0.09,0.03$ and 0.05 for $\mathrm{BN}, \mathrm{AlN}, \mathrm{GaN}$ and $\mathrm{InN}$, respectively. Therefore, the anisotropy of wurtzite AlN crystal is strongest in the calculated materials, but that of $\mathrm{GaN}$ weakest. The conclusion is in agreement qualitatively with Ref. [10] except for BN. 
TABLE III: PHONON FREQUENCIES AT BZ CENTER FOR WURTZITE NITRIDES IN UNITS OF $\mathrm{CM}^{-1}$

\begin{tabular}{|c|c|c|c|c|c|c|c|c|c|}
\hline & & $E_{2}^{l}$ & $B_{1}^{l}$ & $A_{1}(\mathrm{TO})$ & $E_{1}(\mathrm{TO})$ & $E_{2}^{h}$ & $B_{1}^{h}$ & $A_{1}(\mathrm{LO})$ & $E_{1}(\mathrm{LO})$ \\
\hline \multirow[t]{4}{*}{$\mathrm{BN}$} & This work ${ }^{\mathrm{LDA}}$ & 459 & 977 & 1106 & 1137 & 1041 & 1142 & 1305 & 1318 \\
\hline & This work ${ }^{\mathrm{GGA}}$ & 473 & 936 & 997 & 1104 & 945 & 1113 & 1244 & 1267 \\
\hline & Theory $^{[7]}$ & 447 & 918 & 988 & 1024 & 948 & 1179 & 1258 & 1286 \\
\hline & Theory ${ }^{[18]}$ & & & 1006 & 1053 & & & 1258 & 1285 \\
\hline \multirow[t]{5}{*}{ AlN } & This work $\mathrm{k}^{\mathrm{LDA}}$ & 233 & 540 & 597 & 659 & 638 & 679 & 871 & 899 \\
\hline & This work ${ }^{\mathrm{GGA}}$ & 236 & 541 & 604 & 661 & 647 & 716 & 866 & 892 \\
\hline & Theory ${ }^{[10]}$ & 233 & 547 & 614 & 666 & 655 & 719 & 875 & 898 \\
\hline & Experiment ${ }^{[19]}$ & 252 & & 614 & 673 & 660 & & 893 & 916 \\
\hline & Experiment ${ }^{[20]}$ & 241 & & 607 & & 660 & & & 924 \\
\hline \multirow[t]{4}{*}{$\mathrm{GaN}$} & This work ${ }^{\mathrm{LDA}}$ & 147 & 331 & 536 & 554 & 559 & 710 & 731 & 735 \\
\hline & This work ${ }^{\mathrm{GGA}}$ & 137 & 322 & 506 & 524 & 536 & 665 & 703 & 708 \\
\hline & Theory ${ }^{[1]}$ & 137 & 337 & 545 & 563 & 572 & 702 & 736 & 732 \\
\hline & Experiment $^{[21]}$ & 145 & & 533 & 561 & 570 & & 735 & 742 \\
\hline \multirow[t]{5}{*}{$\mathrm{InN}$} & This work ${ }^{\mathrm{LDA}}$ & 79 & 239 & 570 & 598 & 603 & 618 & 687 & 709 \\
\hline & This work ${ }^{\mathrm{GGA}}$ & 85 & 226 & 459 & 481 & 496 & 582 & 596 & 605 \\
\hline & Theory ${ }^{[10]}$ & 83 & 225 & 443 & 467 & 483 & 576 & 586 & 595 \\
\hline & Theory ${ }^{[22]}$ & 87 & 200 & 480 & 476 & 488 & 540 & 580 & 570 \\
\hline & Experiment ${ }^{[23]}$ & & & & & 495 & & 596 & \\
\hline
\end{tabular}

For the sake of comparison, we list the calculated frequencies at $\Gamma$ point in wurtzite BN, AlN, GaN and InN by using the LDA and GGA, as well as other theoretical and experimental values in Table II. It is found that there are some differences between the calculated results by two different exchange-correlation potentials to some extent, even the differences sometimes are very small such as for AIN. The better or worse of the calculated results by two exchange- correlation potentials depends on the specific materials. For example, the results of LDA are more consistent with experimental data and other theoretical results for $\mathrm{AlN}, \mathrm{GaN}$. As for $\mathrm{BN}$ and $\mathrm{InN}$, the GGA potential may be befitting in the calculations. Generally, the frequencies obtained by the GGA are lower than those by LDA especially for InN. In general our calculated results are agreement with existing experi- mental and theoretical data.

\section{CONCLUSION}

We have calculated the phonon dispersion relations for $\mathrm{BN}$, $\mathrm{AlN}, \mathrm{GaN}$ and $\mathrm{InN}$ in the wurtzite structure, by employing both the LDA and the GGA to treat the exchange correlation energy. The results show that the characteristics of phonon frequencies are sensitive to the cation and anion masses. The highest optical phonon frequency at the BZ center decreases, but the phonon band gap increases obviously, with increasing the cation/anion mass ratio. The frequencies calculated with GGA are lower than those with LDA, especially for InN. The GGA is more suitable for AlN and GaN, but the LDA for BN and InN. The anisotropy of wurtzite AlN crystal is stronger than $\mathrm{BN}, \mathrm{GaN}$ and $\mathrm{InN}$. The results are agreement with existing experimental and theoretical data.

\section{ACKNOWLEDGMENT}

This work was supported in part by the PhD Progress Foundation of Higher Education Institutions of China (20111501110003), Natural Science Foundation of Inner Mongolia (No. 2011MS0105) and Talent Development Foundation of Inner Mongolia.

\section{REFERENCES}

[1] V. Ranjan, S. Bin-Omran, D. Sichuga, R. S.Nichols, and L. Bellaiche, "Properties of $\mathrm{GaN} / \mathrm{ScN}$ and $\mathrm{InN} / \mathrm{ScN}$ supperlattices from first principles," Phys. Rev. B, vol. 72, DOI: 10.1103/PhysRevB.72.085315, August 2005.

[2] J. Li, Z. Y. Fan, R. Dahal, M. L. Nakarmi, J. Y. Lin, and H. X. Jiang, "200 nm deep ultraviolet photodetectors based on AlN," Appl. Phys. Lett., vol. 89, 213510, November 2006.

[3] M. Zhang, P. Bhattacharya, and W. Guo, "InGaN/GaN self-organized quantum dot green light emitting diodes with reduced efficiency droop," Appl. Phys. Lett, vol. 97, July 2010.

[4] P. Bhattacharya, M. Zhang, and J. Hinchley, "Tummel injection In $0.25 \mathrm{Ga} 0.75 \mathrm{~N} / \mathrm{GaN}$ quantum dot light-emitting diodes," Appl. Phys. Lett., vol. 97, December 2010.

[5] V. J. Keast, A. J. Scott, M. J. Kappers, C. T. Foxon, and C. J Humphreys, "Electronic structure of GaN and InxGa1-xN measured with electron ener- gy-loss spectroscopy," Phys. Rev. B, vol. 66, September 2002.

[6] A. Siegel, K. Parlinski, and U. D. Wdowik, "Ab initio calculation of structural phase transitions in AlN crystal," Phys. Rev. B, vol. 74, September 2006

[7] H. M. Tütüncü and G. P. Srivastava, "Phonons in zinc-blende and wurtzite phases of GaN, AlN, and BN with the adiabatic bond-charge model," Phys. Rev. B, vol. 62, August 2000.

[8] G. Kaczmarczyk, A. Kaschner, S. Reich, A. Hoffmann, C. Thomsen, D. J. As, A. P. Lima, D. Schikora, and K. Lischka, "Lattice dynamics of hexagonal and cubic InN: Raman-scattering experiments and calculations," Appl. Phys. Lett., vol. 76, February 2000.

[9] K. Shimada, T. Sota, and K. Suzuki, "First-principles study on electronic and elastic properties of BN, AlN, and GaN," J. Appl. Phys., vol. 84, pp. 4951-4958, November 1998. 
[10] C. Bungaro, K. Rapcewicz, and J. Bernholc, "Ab initio phonon dispersions of wurtzite AlN, GaN, and InN," Phys. Rev. B, vol. 61, pp. 6720-6725, March 2000.

[11] F. J. Manjón, D. Errandonea, A. H. Romero, N. Garro, J. Serrano, and M. Kuball, "Lattice dynamics of wurtzite and rocksalt AlN under high pressure: Effect of compression on the crystal anisotropy of wurtzite-type semiconductors," Phys. Rev. B, vol. 77, May 2008.

[12] J. P. Perdew and A. Zunger, "Self-Interaction correction to density-functional approximations for many-electron systems," Phys. Rev. B, vol. 23, pp. 5048-5079, May 1981.

[13] J. P. Perdew, K. Burke, and M. Ernzerhof, "Generalized Gradient Approximation Made Simple," Phys. Rev. Lett., vol. 77, pp. 3865-3868, May 1996

[14] P. Giannozzi et al., "Quantum Espresso: A modular and open-source software project for quantum simulations of materials," J. Phys. Condens. Matter, vol. 21, September 2009.

[15] J. H. Edger, Properties of Group III Nitrides, London, 1994.

[16] A. Wright and J. Nelson, "Explicit treatment of the gallium 3d electrons in GaN using the plane-wave pseudopotential method," Phys. Rev. B, vol. 50, pp. 2159-2165, July 1994.

[17] D. Vogel, P. Krüger, and J. Pollmann, "Structural and electronic properties of group-III nitrides," Phys. Rev. B, vol. 55, pp. 12836-12839, May 1997.

[18] K. Kim, W. R. L. Lambrecholc, and B. Segall, "Elastic constants and related properties of tetrahedrally bonded $\mathrm{BN}, \mathrm{AlN}, \mathrm{GaN}$, and $\mathrm{InN}$," Phys. Rev. B, vol. 53, pp. 16310-16326, June 1996.

[19] L. E. McNeil, M. Grimsditch, and R. H. French, "Vibrational Spectroscopy of Aluminum Nitride," J. Am. Ceram. Soc., vol. 76, pp. 1132-1136, May 1993.

[20] P. Perlin, A. Polian, and T. Suski, "Raman-scattering studies of aluminum nitride at high pressure," Phys. Rev. B, vol. 47, pp. 2874-2877, February 1993

[21] L. Filippedis, H. Siegle, A. Hoffmann, C. Thomsed, K. Karch, and F.Be Chstedt, "Raman Frequencies and Angular Dispersion of Polar Modes in Aluminum Nitride and Gallium Nitride," Phys. Status Solidi B, vol. 198, pp. 621-627, December 1996.
[22] T. Inushima, T. Shiraishi, and V. Y. Davydov. "Phonon structure of InN grown by atomic layer epitaxy," Solid State Commun, vol. 110, pp. 491-495, May 1999.

[23] H. J. Kwon, Y. H. Lee, O. Miki, H. Yamano, and A. Yoshida, "Raman spectra of indium nitride thin films grown by microwave-excited metalorganic vapor phase epitaxy on (0001) sapphire substrates," Appl. Phys. Lett., vol. 69, pp. 937-939, May 1996.

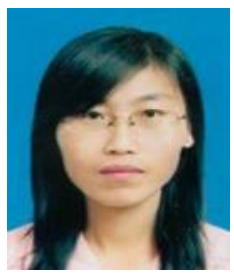

J. J. Zhang obtained her master's degree in 2012 from Inner Mongolia University, majored in theoretical physics. Her research field is the first principle computation of condensed matters.

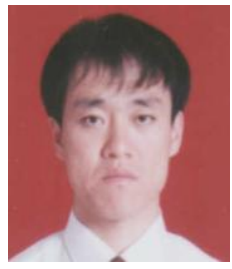

G. J. Zhao received his Ph. D. degree in the area of theoretical physics from Inner Mongolia University, China, in 2003. He is serving as associate professor in Department of Physics Inner Mongolia University from last 8 years. His research interests include Condensed Matter Theory and Computational Physics.

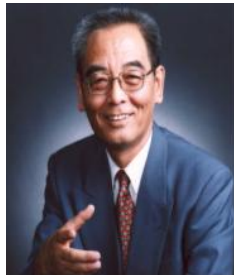

X. X. Liang is the professor in the Department of Physics, Inner Mongolia University, China. His specialization research interests are in Condensed Matter Theory, Computational Physics, and Thermodynamics and Statistical Physics. He has published more that 180 research papers and written 4 books. 